Brit. J. industr. Med., 1953, 10, 161.

\title{
ACUTE POISONING CAUSED BY INGESTION OF ETHYLENE CHLOROHYDRIN*
}

\author{
BY \\ F. BALLOTTA, P. BERTAGNI, and F. M. TROISI \\ From the Institute of Insurance and Legal Medicine of the University of Bologna
}

(RECEIVED FOR PUBLICATION MARCH 10, 1953)

It is thought of interest to report a fatal case of poisoning caused by the ingestion of ethylene chlorohydrin. Cases of poisoning by this substance through the gastro-intestinal tract have not previously been recorded except for two deaths from drinking a solvent mixture containing $25 \%$ of ethylene chlorohydrin (Güthert, 1944).

Ethylene chlorohydrin (glycol chlorohydrin, chloroethyl alcohol), $\mathrm{CH}_{2} \mathrm{Cl}-\mathrm{CH}_{2} \mathrm{OH}$, is a colourless mobile liquid, with an odour resembling that of a mixture of alcohol and ether. The chlorohydrins or halohydrins are derived from dihydric and polyhydric alcohols, in which the halogen atom replaces a hydroxyl group; they are stable compounds. Ethylene chlorohydrin is generally obtained by the action of hypochlorous acid on ethylene.

It has an extensive field of application; it is used as an intermediate product in the preparation of many chemical compounds as an industrial solvent, in the manufacture of a number of insecticides, as a fixative in the colour printing of textiles, for degreasing machinery, and in the treatment of potatoes, particularly sweet potatoes, before sowing in order to hasten germination.

\section{Toxicity}

Ethylene chlorohydrin is among the most toxic of industrial solvents and is absorbed through the lungs, skin, or digestive tract. It has an irritant effect on the eyes and nasal mucosa. Cases of intoxication were reported by Koelsch (1927), Middleton (1930), Dierker and Brown (1944), and Goldblatt and Chiesman (1944), and were caused by inhalation of the vapour and perhaps also by concomitant absorption through the skin in the cases reported by Dierker and

* Translated from the Italian by Dr. L. G. Norman.
Brown and by Middleton. Goldblatt (op. cit.) demonstrated its cumulative toxicity and the greater sensitivity of women and debilitated individuals to its action, and suggested a maximum permissible concentration in atmospheric air of 2 p.p.m.

The symptoms of intoxication consist of nausea, vomiting, headache, drowsiness, vertigo, staggering and incoordinated gait, numbness of the extremities, and visual disturbances. Larger amounts of the poison produce hyperexcitability progressing to delirium; the pulse becomes thin and weak, and the blood pressure extremely low; there is generalized sweating, and finally circulatory collapse and coma. There may be loss of consciousness and spastic contractions of the hands and arms. Erythematous rashes often appear on the arms and trunk; there may also be albuminuria and haematuria. Death occurs from cardiac collapse and pulmonary oedema. After death, in addition to pulmonary oedema with much blood in the alveoli, there is congestion of the cerebral cortex with marked oedema of the cerebral hemispheres ; the liver is very congested and also the kidneys, with haemorrhages and tubular degeneration.

The risk of poisoning at work occurs mainly as a result of the possibility of absorbing ethylene chlorohydrin in the form of vapour, but it may occur also by contact through the skin, when, in certain operations, the clothing of the workers becomes impregnated with it. Its toxicity by the cutaneous route has been found equivalent to that by mouth (Smyth and Carpenter, 1945).

\section{Case Report}

The fatal case of poisoning described here occurred in a young man aged 26 employed at a chemical works, who, while decanting ethylene chlorohydrin from one vessel to another, acted hastily, and instead of using the 
proper emptying pump, took an ordinary rubber tube and aspirated the fluid by mouth, although he well knew the poisonous nature of ethylene chlorohydrin and the container was marked with a label carrying the word "poison" and the skull sign. He felt his mouth fill suddenly with the fluid. He immediately spat it out, then rinsed out his mouth with water, and drank several mouthfuls of water. This took place at about 8.15 a.m. About half an hour later he experienced nausea, retching, and headache, and went to hospital. He was seen immediately on admission, at about 10 a.m., when there were present pallor, retching, and a degree of mental excitability. He was still fully conscious. His general condition was considered to be satisfactory and in no way alarming. There was no congestion or any other appreciable lesion in the mouth or pharynx. He was given a sedative ("luminal "), put to bed, and appeared to become quiet and drowsy. After about two hours, at approximately 12 noon, he jumped out of bed suddenly in the greatest excitement and quickly became almost maniacal : he was swearing, spitting into the air, shouting, and after a sharp and violent struggle with the nurses who were trying to restrain him, he suddenly threw himself down on the bed and almost immediately became comatose, with complete absence of reflexes. There was profuse sweating, and cyanosis followed pallor; the pupils were small and fixed, and all the signs of collapse followed. After hypodermoclysis and the injection of cocarboxylase, there was an apparent improvement and the reflexes reappeared. His condition remained stationary for a time and then suddenly worsened; the pupils again became myotic, while still responding to light, and towards 3.30 p.m. he suddenly experienced generalized tetanic convulsions and the respiration and cardiac contractions ceased, presenting the picture of a bulbar paralysis. Artificial respiration and the intracardiac injection of adrenalin were without effect.

Post-mortem Examination.-The body was of good skeletal formation and excellent muscular development. Rigor mortis was present, and rather marked, dark, hypostatic staining on the back of the body, extending also to the sides. The body was generally well preserved, but over the abdomen a greenish discoloration was already appearing. The natural orifices were dry. There was no particular odour from the body.

Head.-The brain and meninges were markedly congested, particularly when examining the meningeal linings. The meninges were free from any lesions or signs of disease, but the brain was translucent on account of moderate oedema. Section of the various parts of the brain did not reveal even punctiform haemorrhages or softening.

Neck and Thorax.-There were no signs of irritation or other lesion in the posterior parts of the mouth, the larynx, pharynx, or upper part of the oesophagus. The thyroid was normal in size and appearance. The thymus, of a reddish colour, was rather large for the age of the individual and very congested ( $35.5 \mathrm{~g}$.).
The pleura was smooth and shiny, as also was the pericardium; there was a small quantity of yellow fluid in the pericardial sac. The heart was of normal size in relation to the heavily built body and was anatomically normal. On the posterior aspect of the heart were numerous punctate haemorrhages (Tardieu's spots).

The lungs were very bulky. From the cut surfaces exuded abundant quantities of reddish, frothy fluid, and from the mouths of the cut vessels poured dark, thick blood. The red, frothy fluid filled not only the lungs but the lower air passage also. A number of Tardieu's spots were noted on the surface of the lungs. The frothy fluid from the lungs was acid in reaction.

Abdomen.-The lower part of the oesophagus showed no irritation or signs of any other lesion. The stomach contained about $10 \mathrm{ml}$. of reddish turbid fluid with the characteristic odour of normal gastric contents. The gastric mucosa showed many superficial haemorrhagic effusions. The liver was very congested; its size, consistence, and colouring were normal. The spleen was a little enlarged, with somewhat tense capsule. The left kidney was much reduced in size, with a very uneven surface, and was covered with a thickened capsule which was very closely adherent to the underlying parenchyma. The parenchyma was reduced to a few trabeculae, and between these were cavities, empty or nearly so, surrounded by a very thick, whitish fibrous tissue lining. The ureter of this kidney was much narrowed and transformed into a cord-like structure. The left suprarenal was also much smaller than normal, but was of normal shape and showed no appreciable macroscopic changes. The right kidney was almost double a normal kidney in volume; its capsule peeled easily. The relative amounts of cortex and medulla appeared to be normal. The size of the ureter was adequate for the size of the kidney. The right suprarenal was also larger than the left. The bladder contained a few cubic centimetres of turbid urine which had a neutral reaction.

Histological examination of the pharynx did not reveal any evidence of irritation. Hyperaemia was generally observed in the preparations of the various organs. In the right kidney and the functioning part of the left were seen, in addition to congestion, haemorrhagic extravasations both in and surrounding the tubules (Figs. 1 and 2) and a moderate oedema of the glomerular and tubular epithelium.

Estimation of Ethylene Chlorohydrin in Blood and Urine.-For the determination of ethylene chlorohydrin in the blood and urine, because only a small quantity of urine was available, the micromethod of Widmark was used. A simplified Widmark apparatus was used for the determinations, as described by Smith and Glaister (1939), using the same technique for ethylene chlorohydrin and taking care to carry out the distillation at about $130^{\circ} \mathrm{C}$. in order to ensure the complete oxidation of the ethylene chlorohydrin. With this method a concentration of ethylene chlorohydrin of $27.89 \mathrm{mg}$. \% was found in the blood and 

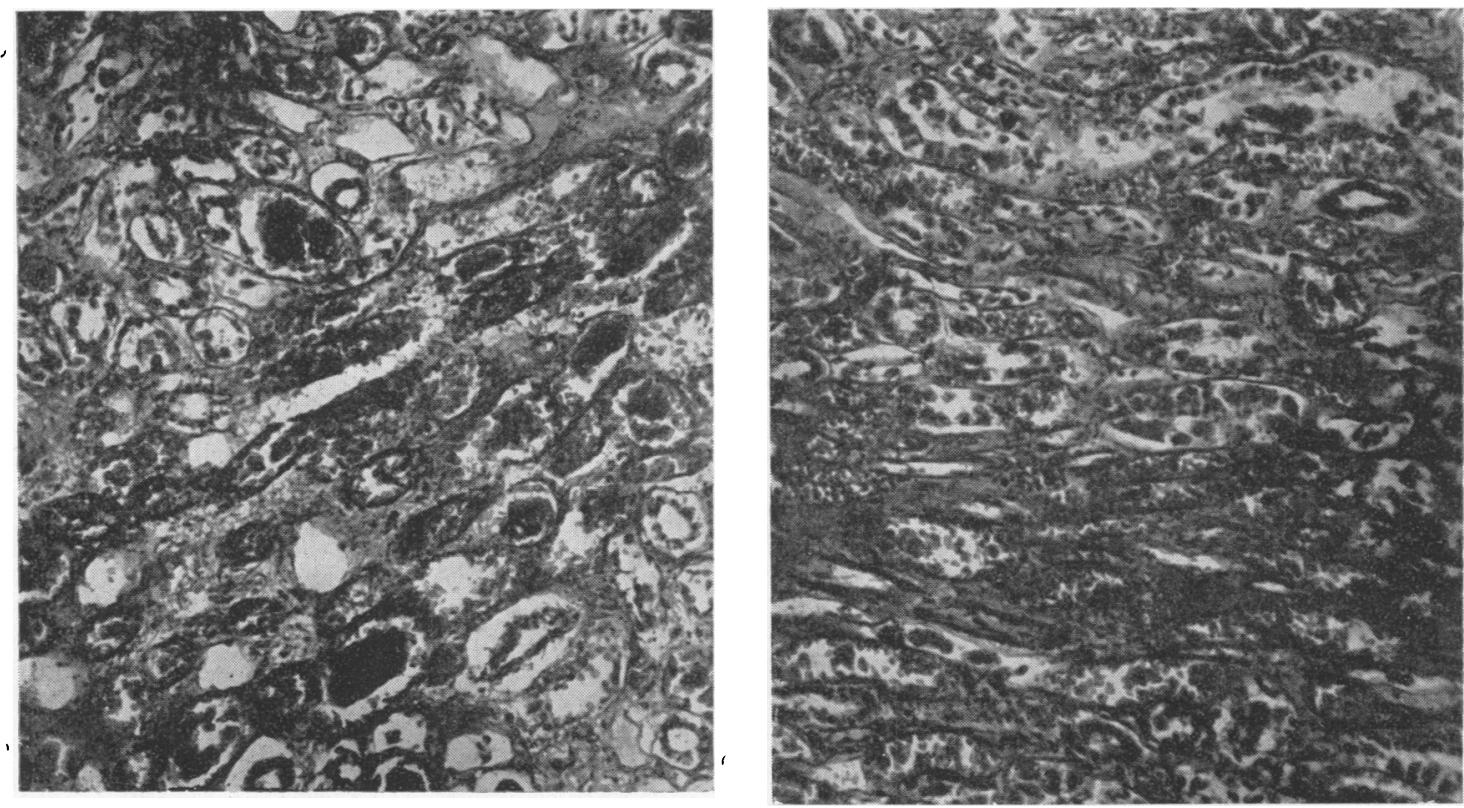

FIGS. 1 and 2.-Sections of kidney showing haemorrhagic extravasations both in and surrounding the tubules and oedema of the glomerular and tubular epithelium.

$80.49 \mathrm{mg} . \%$ in the urine. The difference in concentration of ethylene chlorohydrin between blood and urine is similar to that found with ethyl alcohol.

The clinical symptomatology and the pathological and histological examination suggest that in this case death occurred through cardiac and circulatory collapse, pulmonary oedema, and involvement of the central nervous system, and the toxic picture resembled that of acute ethyl alcohol poisoning. The toxic action was particularly effective on the blood vessels, causing an intense hyperaemia of all organs and irritation of the bulbar centres, terminating in bulbar paralysis.

\section{Summary}

A fatal case of poisoning caused by the ingestion of a small quantity of ethylene chlorohydrin is described. Death occurred after seven hours, the symptoms consisting of nausea, retching, head- ache, excitability, delirium, coma, and collapse. Death was apparently due to cardiac and respiratory collapse. Post-mortem and histological examination showed no changes in the mouth and pharynx ; hyperaemia of various organs, particularly of the brain and meninges, and of the liver; marked oedema of the lungs ; and extravasation of blood, congestion, and oedema, in and around the renal tubules.

\section{REFERENCES}

Dierker, H. and Brown, P. G. (1944). J. industr. Hyg., 26, 277. Goldblatt, M. W., and Chiesman, W. E. (1944). British Journal of Industrial Medicine, 1, 207.

Industrial Medicine, 1, 207.

Koelsch, F. (1927). Zbl. GewHyg., (n.s. 4) 14, 312.

Middleton, E. L. (1930). J. industr. Hyg., 12, 265.

Smith, S., and Glaister, J. (1939). Recent Advances in Forensic Medicine, 2nd ed. Churchill, London.

Smyth, H. F., Jr., and Carpenter, C. P. (1945) J. industr. Hyg., 